\section{LUIS CRESPÍ JAUME, CIENTÍFICO DE LA JUNTA PARA AMPLIACIÓN DE ESTUDIOS Y CATEDRÁTICO DE AGRICULTURA DEL INSTITUTO-ESCUELA}

\author{
Carmen Masip Hidalgo \\ IES Isabel la Católica (Madrid) \\ C/ Sirio, n.o 8 dcha, 7.o A. 28007 Madrid
}

carmenmasip@terra.es

\section{LUIS CRESPÍ JAUME, A SCIENTIST OF THE JUNTA PARA AMPLIACIÓN DE ESTUDIOS AND PROFESSOR OF AGRICULTURE OF THE INSTITUTO-ESCUELA}

ABSTRACT: This article about Luis Crespi Jaume (Madrid, 18891963) aims to recall his teaching work as Agriculture Professor at "Madrid Instituto-Escuela" and its stage as scientist linked to "Junta para Ampliación de Estudios "(JAE). This following text focuses on his teaching practice which was based on the analysis of the didactic planning in the field of Agriculture that he developed, the methodology applied, the scientific materials used in the class and laboratory sessions, the class and laboratory students' notebooks and finally the books that they consulted for their classworks.

KEY WORDS: "Junta para Ampliación de Estudios" (JAE); "InstitutoEscuela"; Crespi; history of education; agricultural education; school notebooks; Vavilov; National Museum of Natural Sciences (MNCN); Royal Botanical Garden (RJB); Royal Spanish Society of Natural History (RSEHN).

\section{La etapa de formación de Luis Crespi}

El ambiente familiar en el que vivió los primeros años de su vida Luis Crespi Jaume fue determinante para su vocación y formación como hombre de ciencia. Su padre, Antonio Crespi Mas, de origen mallorquín, era doctor en Farmacia y en Ciencias Naturales, catedrático de Agricultura y Técnica Agrícola e Industrial en el Instituto General y Técnico de Pontevedra y experto en Botánica. Luis Crespí compartió con sus hermanos Miguel, Andrés, Antonio y Carlos el amor por la naturaleza que el padre les había inculcado desde la infancia. Cuando terminaron el bachillerato y entraron en la universidad, la pasión por el mundo natural habría de conducir a los hermanos Crespí al estudio de diversas disciplinas científico-técnicas. Luis estudió Ciencias Naturales; Miquel, Química; Andrés, Dibujo; Antonio y Carlos, Topografía. Pocos años después de su paso por la universidad, los tres primeros, incorporados ya a la docen-
RESUMEN: Este artículo sobre Luis Crespi Jaume (Madrid, 18891963) pretende recordar su labor pedagógica como catedrático de Agricultura del Instituto-Escuela de Madrid y su etapa como cientifico vinculado a la Junta para Ampliación de Estudios (JAE). Nuestro comentario sobre su práctica docente se basa en el análisis de la programación de la materia de Agricultura que él mismo elaboró, en la metodología que aplicó, en los materiales científicos utilizados en el aula y en el laboratorio, en los cuadernos de clase y de prácticas de sus alumnos y en los libros que éstos consultaban para sus trabajos.

PALABRAS CLAVE: Junta para Ampliación de Estudios (JAE); Instituto-Escuela; Crespi; historia de la educación; enseñanza de la agricultura; cuadernos escolares; Vavílov; Museo Nacional de Ciencias Naturales (MNCN); Real Jardín Botánico (RJB); Real Sociedad Española de Historia Natural (RSEHN).

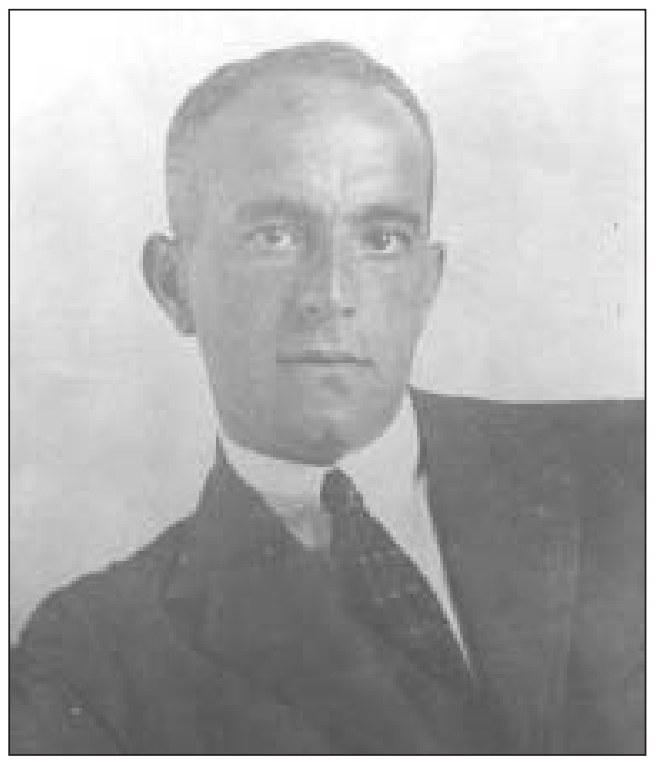

Luis Crespí Jaume 
cia, llegaron a disfrutar de las pensiones que concedia la Junta para Ampliación de Estudios (JAE) para mejorar la formación en el extranjero, en tanto que Carlos participó en la famosa Comisión Científica a Ifni auspiciada también por la Junta.

Luis Crespí ingresó en el instituto de Pontevedra en 1899 para estudiar el bachillerato y obtuvo el grado en marzo de 1906. Después se matriculó en la Universidad de Santiago de Compostela para cursar materias de Física, Química, Mineralogía, Botánica y Zoología (Fraga Vázquez, 1995, 118) y más tarde se trasladó a Madrid, en cuya Universidad Central se licenció en Ciencias Naturales (Crespi; González Bueno, 1990, 341-345; Fraga, 1995, 118).

Una vez concluidos sus estudios universitarios, Crespi se interesó inicialmente por cuestiones agrícolas y de fisiología vegetal que le aproximaron a la ecología vegetal en su vertiente aplicada (Casado de Otaola, 2000, 258). Su trabajo como científico comenzó en el Museo Nacional de Ciencias Naturales (MNCN). En 1912, Crespí realizó en el Museo una serie de experiencias con el maíz para contrastar las hipótesis de Blaringhem sobre el transformismo aplicado en agricultura del maíz.

El 29 de octubre de 1914 solicitó una beca para estudiar Geología agrícola en el $\mathrm{MNCN}^{1}$, donde trabajó como ayudante del laboratorio de Geología del Museo. En este laboratorio, bajo la dirección de Hernández Pacheco, estudió los terrenos geológicos de la Península lbérica en relación con la Agricultura. Asistió a prácticas de análisis de tierras en los laboratorios del MNCN y estudió hongos parásitos en el laboratorio de Micología bajo la dirección del botánico González Fragoso, para cuyas publicaciones además realizó dibujos.

Después de ser admitido en la Real Sociedad Española de Historia Natural (RSEHN), en enero de 1915, en mayo de este mismo año publicó los resultados de sus investigaciones sobre el maíz en Las anomalías florales del maíz y los trabajos de Blaringhen. Durante el año 1915 y el primer trimestre de $1916^{2}$, también trabajó como becario en un curso de Zoología.

Su carrera profesional como docente comenzó a los 26 años, cuando en 1916 ganó por oposición la cátedra de Agricultura de instituto. Sus primeros destinos fueron los institutos de Lugo y Pontevedra. Pero la tarea docente no le impidió trabajar en otros ámbitos. En 1917, Luis Crespí y Juan Dantín Cereceda colaboraron en el curso de Fisiología vegetal que organizó la JAE en el Real Jardín Botánico de Madrid.

Siendo todavía catedrático en Lugo, Crespi obtuvo una primera pensión de la JAE en el curso 1917-18 para una estancia de nueve meses en Toulouse (Francia), donde asistió a los cursos y trabajos prácticos de Fisiología vegetal dirigidos por Leclerc du Sablon, asi como a los de Patología Agrícola dirigidos por Prunet. Bajo la dirección de Leclerc du Sablon, realizó experiencias sobre la evolución de los hidratos de carbono contenidos en las semillas de las leguminosas durante la germinación. Este trabajo de investigación lo continuó luego en el laboratorio de Fisiología vegetal creado por la JAE en Madrid ${ }^{3}$.

\section{Luis Crespí, catedrático del Instituto-Escuela Y CIENTífico de la Junta PARA Ampliación dE Estudios e Investigaciones Cientificas (JAE)}

El 13 de julio de 1918, Luis Crespí fue nombrado provisionalmente catedrático de Agricultura del Instituto-Escuela, que aquel mismo año la JAE puso en funcionamiento como un ensayo pedagógico de segunda enseñanza. Desde entonces, Crespí ejerció la docencia en el Instituto-Escuela hasta que este centro tuvo que cesar en su actividad en el verano de 1936 a causa de la guerra civil. Es importante reseñar que, para ocupar la plaza de catedrático de Agricultura del Instituto-Escuela, la JAE consideró como méritos algunos de los trabajos de investigación que Crespí había llevado a cabo en el Museo Nacional de Ciencias Naturales y los que había realizado como pensionado de la JAE en Toulouse.

En enero de 1920, la JAE invitó al profesor Lewis Knudson, de la Universidad de Cornell (Nueva York) a dar un curso práctico de Fisiología vegetal en el Jardín Botánico de Madrid. Ramón y Cajal, como presidente de la JAE, en una carta remitida al director del Jardín Botánico, consideraba a Knudson una de las personalidades más sólidas en aqueIla especialidad y le solicitaba un permiso para que durante el mes de febrero se dieran unas clases preparatorias a los que iban a asistir al curso. En la carta, le comunicaba 
que el profesor del Instituto-Escuela Luis Crespí había sido encargado de este curso de preparación, de acuerdo con las instrucciones dadas por el profesor Knudson. Este y otros cursos realizados por los pensionados de la JAE en el extranjero fueron el germen del laboratorio de Fisiología vegetal que poco después puso en marcha la JAE (Baratas, 1998, 160).

Aunque en 1922 Crespí fue nombrado catedrático numerario de Agricultura del Instituto General y Técnico de Guadalajara ${ }^{4}$, continuó su labor docente en el Instituto-Escuela ${ }^{5}$. Aquel mismo año publicó en la colección Catecismos del Agricultor y del Ganadero La soja y su cultivo en España, en la que describe el cultivo de la soja, sus aplicaciones, la distribución geográfica de su cultivo en España y en el resto del mundo, y su aparición en Europa, en 1790. Entre otros aspectos, comenta los ensayos que se hicieron para el cultivo de la soja hasta 1920 en España y, de paso, recuerda que su padre fue de los primeros en llevarlos a cabo.

En 1924, Crespí obtuvo una segunda pensión de la JAE para ampliar su formación científica en el extranjero durante un año. Primero viajó a Portugal, donde estudió los líquenes bajo la dirección del profesor Gonzalo Sampaio, de la Universidad de Oporto, con el que seguirá colaborando en los años siguientes. En Portugal permaneció en Braga, por ser la ciudad más adecuada para realizar las excursiones al campo. Desde allí, se trasladó a Francia y Bélgica para conocer el funcionamiento de la enseñanza agrícola en los principales establecimientos docentes ${ }^{6}$. Pero el 31 de junio de 1925, tuvo que interrumpir su estancia europea y regresar a España a consecuencia de una desgracia familiar.

Durante las vacaciones de verano y de Navidad de 1925, realizó varias excursiones liquenológicas con su cuñado Miguel Rodríguez, aficionado a la botánica, por la provincia de Pontevedra, y formó una colección que donó al Museo Nacional de Ciencias Naturales ${ }^{7}$. Entretanto, había retomado en octubre la pensión interrumpida en el mes de junio. Durante los cuatro meses de pensionado que le quedaban, se dedicó con Gonzalo Sampaio a determinar los materiales liquenológicos recolectados en las excursiones que había hecho con su cuñado. Poco después, Crespí y Sampaio publicaron de manera conjunta Los líquenes de la provincia de Pontevedra (1927), en el que dieron a conocer varias especies nuevas.
En 1927 viajó a España el famoso genético ruso Nikolái Ivánovich Vavílov (Moscú, 1887-Saratov, 1943), director del Instituto para la Producción Vegetal de Leningrado. Vavílov estaba investigando sobre la inmunidad de las plantas, y realizaba una serie de expediciones botánicoagronómicas por todo el mundo para desarrollar su teoría de los centros de origen de algunas plantas cultivadas y ampliar el mayor banco de semillas del mundo de la época en su instituto de Leningrado. Luis Crespí e Ignacio Bolívar, el director del Museo Nacional de Ciencias Naturales, colaboraron en la organización de la llegada de Vavílov a España ${ }^{8}$; se hicieron cargo en 1926 de tramitar la solicitud del permiso de entrada a España del famoso agrónomo. Pero Vavílov demoró el viaje hasta 1927, pues se desplazó antes a Abisinia (hoy Etiopía), porque la cosecha se recogía en aquella época del año, mientras que en España comenzaba más tarde, en abril y mayo. Tras el retraso de Vavílov, Crespí y Bolivar se encargaron de gestionar la renovación de su permiso de entrada.

En 1926, la JAE comisionó a Crespí para dirigir las Misiones culturales de Galicia con el fin de realizar estudios botánicos y folkloristas (Casado de Otaola, 2000, 347; Crespí, 1929; González Bueno, 1990, 344; Fraga, 1995, 118) en la sierra de los Ancares (Lugo) y en las de Queija e Invernadeiro (Orense). De esta expedición formaron parte, además de Crespí, Gonzalo Sampaio, Luis Iglesias (profesor de la Universidad de Santiago), el propio Vavílov y otros naturalistas. Crespí informó y dio cuenta de los gastos de las Misiones culturales de Galicia en una carta que dirigió a Ignacio Bolivar'. En 1929, Crespí e Iglesias publicaron Los prados de las regiones media y montaña de Galicia. Este trabajo muestra una clara orientación ecológica de Crespí (Casado de Otaola, 2000, 258), el cual estudia la ganadería, el sistema de pastoreo y los prados para la producción de heno, analizando la composición florística, evaluando su estado y proponiendo una serie de reformas para un mayor rendimiento.

Por este tiempo, Crespí, como catedrático de Agricultura del Instituto-Escuela, participó con Notas sobre una geografía botánica en Galicia (1929), en el libro homenaje a Ignacio Bolivar publicado en las Memorias de la Sociedad Española de Historia Natural. En su colaboración, ofrece un avance de la geografía botánico-agrícola de la sierra de los Ancares e Invernadeiro, como resultado de los estudios realizados en ella.

ARBOR Vol. 187749 mayo-junio [2011] 501-511 ISSN: 0210-1963

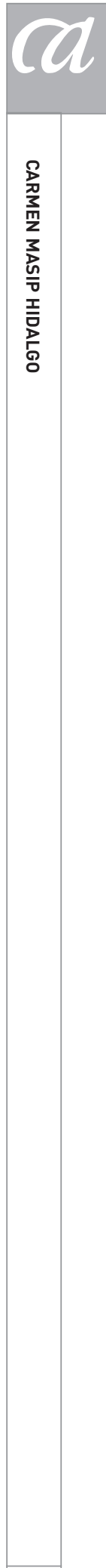

503 
Desde el primer momento, se estableció una estrecha relación entre Vavílov y Crespí, de la que surge su interés por la genética y el origen de las plantas cultivadas (Casado de Otaola, 2000, 258). Prueba de la amistad que ambos forjaron es que Luis Crespí le dedicó a Vavilov su trabajo Contribuciones al folklore gallego, editado en 1929 por la JAE Misiones culturales de Galicia (Fraga; Gurriarán, 2007,194), en el que estudia las pallozas desde Cebrero hasta Piornedo, el hórreo, el rodicio de un molino, los aperos de labranza y los distintos tipos de arados romanos.

A su vez, Vavilov mencionó numerosas veces a Crespí en Expedición por España (1931), el informe del viaje que realizó en 1927 por nuestro país. En este informe hace alusión al agradable recibimiento que le dispensaron en España, "especialmente el director del Museo de Historia Natural, el conocido entomólogo profesor Ignacio Bolivar, el hijo de éste y el botánico Crespi". Después de completar las investigaciones en el sur de de España, Vavílov fue, a mediados del mes de julio de 1927, a la ciudad de León con la intención de iniciar los estudios de las plantas cultivadas de Asturias y Galicia; en su informe Vavílov agradece la enorme ayuda de Crespí, con quien había hecho docenas de paquetes con semillas y espigas para expedir a Leningrado, prolongando la jornada de trabajo incluso por la noche. La relación de amistad y colaboración entre Crespí y Vavílov se mantuvo hasta el final de la II República. Siendo Crespí su vicepresidente, la Sociedad Española de Historia Natural recibió una invitación para asistir en Leningrado, el 16 de febrero de 1935, a la fiesta organizada por el Instituto de Producción Vegetal, con el propósito de rendir homenaje a Vavílov, su director.

El 16 de abril de 1930, Crespí obtuvo el nombramiento definitivo como catedrático del Instituto-Escuela ${ }^{10}$, y en marzo de 1933 la JAE lo nombró jefe del recién creado Laboratorio de Ecología del Museo Nacional de Ciencias Naturales (Casado de Otaola, 2000, 258; Crespí y González Bueno, 1990, 344; Archivo JAE Edad Plata). Compatibilizó este cargo con la enseñanza en el Instituto-Escuela $y_{\text {, }}$ cuando este centro cesó en su actividad en el verano de 1936, siguió dando clases en el instituto Pérez Galdós y en el San Isidro, hasta el final de la guerra civil ${ }^{11}$. Fue también en 1933 cuando el Ministerio de Instrucción Pública y Bellas Artes lo nombró secretario de la Junta de Sustitución de la Segunda Enseñanza de las Congregaciones Religiosas. Esta Junta tenía no sólo la misión de sustituir al profesora- do religioso por uno laico, sino también la de inspeccionar y dotar a los centros educativos. Por ello, Crespí solicitó al Museo Nacional de Ciencias Naturales que preparara y enviara colecciones de animales, plantas y minerales a los laboratorios de Ciencias Naturales y Agricultura de los nuevos colegios y centros de enseñanza ${ }^{12}$.

Crespí pertenecía a la segunda generación de micólogos que trabajó con Blas Lázaro Ibiza, el primer catedrático de Botánica de la Facultad de Farmacia de la Universidad Central, y en los años treinta aparece como botánico colaborador y recolector en la obra de otros tres micólogos importantes, Luis M. Unamuno, González Fragoso y Emilio Guinea. Unamuno, como reconocimiento a la labor de su compañero y colaborador de la JAE y del Real Jardín Botánico, le dedicó a Crespí un nuevo liquen, el taxón Puccinia crespiana (1935) (González Bueno,1993). El fondo botánico de Emilio Guinea López, que fue profesor aspirante de Ciencias Naturales en el Instituto-Escuela Sección Retiro durante el curso 1932-1933, se conserva en el Real Jardín Botánico y cuenta con 39 fichas y dibujos de distintos líquenes que se deben a la mano de Crespí.

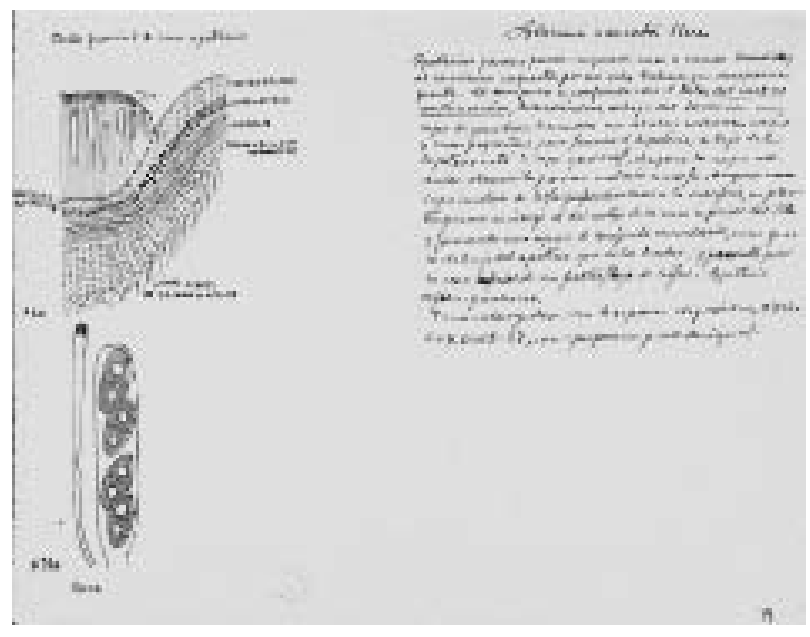

Ficha de un liquen realizada por Luis Crespí Jaume. Archivo del Real Jardín Botánico.

Después de la guerra civil, Crespí sufrió represalias, y fue depurado por las autoridades franquistas, con suspensión de empleo y sueldo e inhabilitación para la docencia durante catorce años. A lo largo de todo este tiempo, trabajó como administrativo en los Laboratorios Promesa de 
Madrid (Casado de Otaola, 2000, 347; Crespí; González Bueno, 1990, 344). Cuando Joaquín Ruiz-Giménez era director general de Enseñanzas Medias del Ministerio de Educación, fue reintegrado a su cátedra el 3 de abril de $1954^{13}$, aunque sin levantarle la sanción de inhabilitación por tres años más para el ejercicio de cargos directivos y de confianza. En los años siguientes, ejerció la docencia en el instituto San Isidro. Después de la guerra civil, las represalias, la depuración y la inhabilitación a que se vio sometido fueron las causas de que la labor de Crespí como investigador acabara en 1938. Murió el 12 de febrero de 1963 en Madrid.

En 1999, Víctor J. Rico le dedica un nuevo liquen, Asplicilia crespiana y señala con tal motivo: "Esta especie es dedicada a Luis Crespí Jaume (Madrid 1889-1963), quien la recolectó durante 1920" (Rico, 1999).

\section{La enseñanza de la Agricultura en el Instituto-Escuela}

En el Instituto-Escuela, la enseñanza de la Agricultura, como las del resto de las materias, era una enseñanza innovadora y rigurosa, pedagógica y científicamente actualizada, que estimulaba la participación activa de los alumnos en las clases, en los laboratorios, en los trabajos de campo y en las excursiones. Se trataba de dar un enfoque metodológico nuevo en la enseñanza del medio natural, con ideas precursoras de la ecología, como se hacía en Europa (Bernal; Comas, 2006), fomentando en los alumnos el amor a la naturaleza y la sensibilidad en su conservación.

De acuerdo con el plan de estudios del Instituto-Escuela, la materia de Agricultura se impartía inicialmente en $4 .{ }^{\circ}$ curso y después, tras un cambio en el plan, en $5 .{ }^{\circ}$ de Bachillerato. Se le dedicaban dos clases semanales de hora y media cada una y el responsable de programar sus contenidos fue Crespí.

En la programación de la materia, Crespí hace una relación de los contenidos que va a desarrollar en el curso. Sin pretender ser exhaustivos por nuestra parte, para él tienen especial importancia los contenidos relativos a las cuestiones teóricas o a los fundamentos de la Agricultu- ra; la planta como productora de sustancias orgánicas; la multiplicación, variabilidad, variedad y forma de los vegetales; las modificaciones de éstos producidas por el suelo, el hombre y el clima; la tierra vegetal; los abonos; diferentes tipos de cultivos y el ganado.

El enfoque pedagógico de la materia de Agricultura escogido por Crespí distaba mucho del puramente teórico y memorístico tradicional, que era el empleado generalmente fuera del Instituto-Escuela. Formaba parte de su metodología que los alumnos ensayaran las diversas técnicas agrícolas explicadas en el aula e hicieran prácticas en el laboratorio. Las visitas a museos y centros de producción, así como las excursiones al campo, eran actividades formativas muy importantes en el Instituto-Escuela. En relación con la Agricultura, los alumnos realizaban visitas a centros agrícolas de Floricultura, a La Granja y Escuela Central de Agricultura o a las estufas del Jardín Botánico, además de otras excursiones de carácter interdisciplinar ${ }^{14}$. Con los trabajos en clase, las visitas a centros agrícolas y las prácticas en el campo, Crespí pretendia fomentar entre ellos la afición a la vida campesina y el gusto por el cuidado de las plantas y de los animales útiles.

En el Instituto-Escuela, a diferencia de los institutos oficiales, no se utilizaban como recurso didáctico los libros de textos tradicionales, por estar desfasados, pero en los cursos superiores los alumnos disponían de manuales y libros de consulta en español y en otros idiomas (alemán, francés e inglés) para realizar determinados trabajos, como Chimie Agricole, Chimie végétale, de G. André; Arboricultura frutière, de L. Bussard y G. Duval; Les Légumes et les Fruits, de J. De Brevans; y The informations reader n. ${ }^{\circ}$. Foods and Beverages, de la Boston School Series, entre otros ${ }^{15}$.

Con el fin de que se familiarizaran con los tecnicismos agrícolas más frecuentes y pudieran leer con provecho libros de Agricultura, Crespí recomendaba distintas lecturas, particularmente los Catecismos del Agricultor, por la variedad y sencillez de los temas que trataban.

Para el aprendizaje de las distintas materias, en el Instituto-Escuela se implantaron los cuadernos de clase, elaborados por los propios alumnos y corregidos por el profesor. En estos cuadernos, los alumnos recogían las intervenciones del profesor, las prácticas del laboratorio y las excursiones

ARBOR Vol. 187749 mayo-junio [2011] 501-511 ISSN: 0210-1963 las cosechas; las características agrícolas de España; los

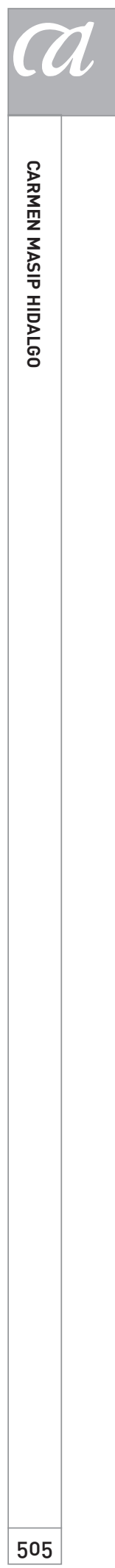

doi: 10.3989/arbor.2011.749n3004 
(Martínez Alfaro, 2009, 246-252). Los alumnos utilizaban los cuadernos como una herramienta básica de trabajo (Sánchez de Andrés, 2007, 421-428). Para el aprendizaje de la Agricultura, los alumnos utilizaban dos tipos de cuaderno: uno para anotar los conocimientos impartidos por el profesor en el aula y otro más específico para las prácticas de laboratorio. Algunos de estos cuadernos aún se conservan ${ }^{16}$.

Como trabajos prácticos de campo, los alumnos preparaban el terreno y la siembra y cultivaban gran número y variedad de plantas en los jardines del Instituto-Escuela. Con algunas plantas seguian todas las fases de su crecimiento y con otras, solamente las primeras, por la interrupción de las vacaciones. El resto de prácticas abarcaba la plantación de árboles, ejercicios de poda, riego, acodos, estacas y trasplantes.

Pero los alumnos no sólo realizaban prácticas en el campo. En las clases resolvían problemas agrícolas y en el laboratorio completaban su formación con experiencias de Fisiología vegetal sobre transpiración, extracción de la clorofila, geotropismos, nutrición mineral de las plantas verdes o la preparación de líquidos anticriptogámicos. En los cuadernos de prácticas, se puede advertir el objetivo que se marcó Crespí para impartir un modelo de enseñanza aplicada de la Agricultura. Por ejemplo, una parte de las experiencias de laboratorio las dedicaba al estudio del mosto del vino desde una perspectiva teórico-práctica e interdisciplinar. A partir de la práctica de laboratorio, los alumnos, basándose en estudios y técnicas diversas de elaboración, completaban su conocimiento sobre los distintos tipos de vino y la fitopatología de la vid. Los alumnos comenzaban sus prácticas observando y dibujando (Cuaderno de Prácticas) el aspecto de la uva y con las yemas de los dedos prensaban la uva sin escobajos, con lo que obtenían un líquido dulce, traslúcido pero no transparente, el mosto. A continuación, prensaban los hollejos con una prensa que aún se conserva ${ }^{17}$ y obtenían otro mosto. Luego, mezclaban ambos líquidos en un erlenmeyer. En el líquido resultante, median la densidad del mosto con un densímetro y deducían los litros de alcohol y la cantidad de azúcar por hectolitro de mosto. Añadían después al líquido los hollejos para variar el color, entre amoratado y rojo, gracias a los taninos, que están fundamentalmente en los hollejos. Por último, resolvían problemas de estequiometría para averiguar la cantidad de alcohol a partir

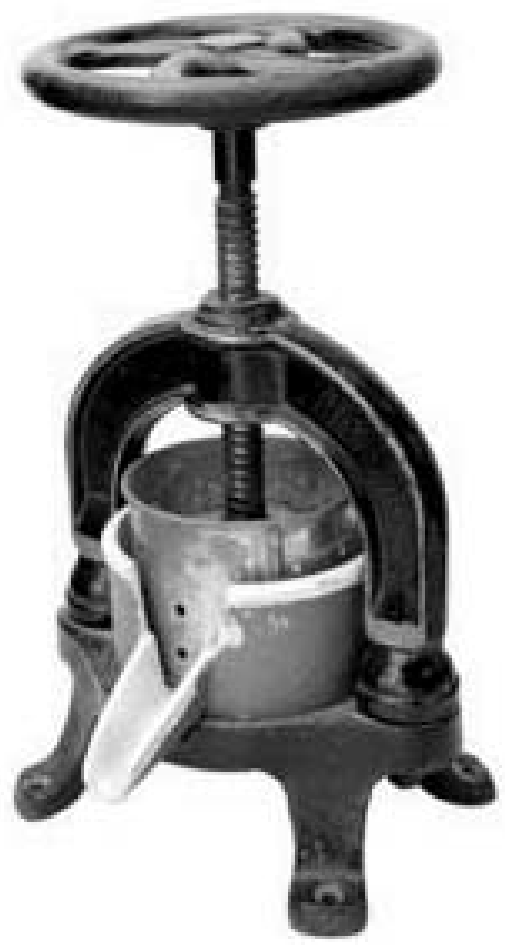

Prensa utilizada en prácticas de Agricultura. Instituto-Escuela. Sección Retiro.

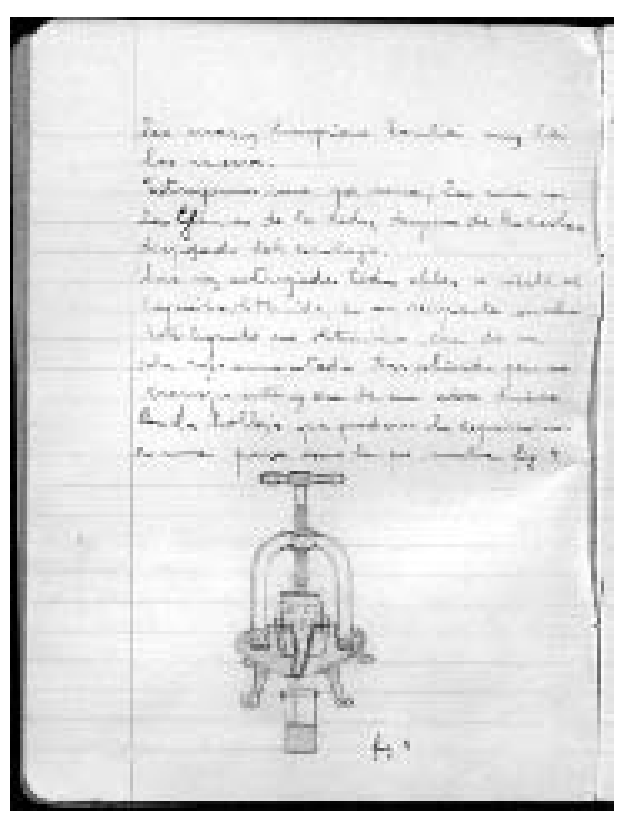

Cuaderno de Prácticas de Agricultura del alumno Javier Cabañas. Instituto-Escuela. Sección Retiro. 
de la reacción de fermentación del azúcar y analizaban la composición de la pulpa. En el cuaderno específico para prácticas, los alumnos dibujaban, explicaban todo el proceso, anotaban los resultados de los análisis y estudiaban el color y la turbidez del vino. Posteriormente, concluian el trabajo con un estudio teórico sobre la fermentación por Saccharomyces cerevisiae, estableciendo diferencias entre vinos dulces, espumosos, etc.

Por ser importante, los cuadernos recogen la geografía botánica de algunos cultivos en relación con la distribución de las bebidas alcohólicas según el clima. Así, los alumnos señalan que la distribución del vino está condicionada a zonas templadas, porque éstas permiten la madurez de las vides y la humedad es un factor limitante. El conocimiento de las zonas templadas y la humedad les sirve para comparar la distribución climática del vino con la distribución climática de otras bebidas alcohólicas como la sidra y la cebada. Por todas estas observaciones, los alumnos saben que en el Cantábrico, por ser la costa muy húmeda, no se produce la vid y el vino es sustituido por la sidra, una bebida alcohólica elaborada a partir de la manzana. Luego anotan que el vino se produce hasta el norte de Francia, donde vuelve a predominar la sidra; y más al norte, en los Países Bajos y Alemania, se da la cebada, con la que se fabrica la cerveza. En los cuadernos se refleja que en los climas cálidos y en algunos países africanos, se obtienen bebidas alcohólicas de la fermentación del jugo de la palmera y de la pita (Cuaderno de Prácticas).

En patología vegetal, los alumnos estudiaban las enfermedades parasitarias de la vid (Cuadernos III-IV) como el mildíu, la clorosis o la filoxera -de la que observaban preparaciones microscópicas de los huevos y de la ninfa (n. de catálogo 630)-, y los métodos de lucha para combatirlas. Para completar el estudio del vino, también debían consultar libros como Cuidados del vino en el primer año ${ }^{18}$, de la ya citada colección Catecismo del Agricultor, en la que aprendían la fermentación lenta, los rehenchidos o rellenos, el tapado de los envases, los azufrados y los trasiegos para separar las lías o heces de los vinos que se han formado.

En la clase, los trabajos prácticos que Crespí programaba tenían por objeto la resolución de problemas agrícolas y el reconocimiento de tierras de labor, abonos y semillas (JAE, 1925, 286). Los alumnos estudiaban la colección de semillas -parte de la cual aún se conserva ( $n .^{\circ}$ de catálogo 176-178)- y ensayaban germinaciones. En un germinador y durante doce horas, ponian en remojo semillas de uvas, altramuces, granos de trigo, avena, etc. En el cuaderno, dibujaban luego los procesos de germinación, el fruto y las semillas de la remolacha, del girasol y del algodón. De los cereales, dibujaban la estructura, la disposición de la espiguilla y el diagrama floral del trigo (Cuaderno de Prácticas y Cuaderno I). Lo mismo cabe decir en relación con un gran número de plantas agrícolas, como la patata, el naranjo o el limonero (Cuaderno I). Consignaban detalles para su reconocimiento y reflejaban las características de su cultivo y aprovechamiento. También analizaban la importancia de la selección semillas, su desinfección, el poder germinativo y el valor agrícola (resistencia a heladas, calor, etc.).

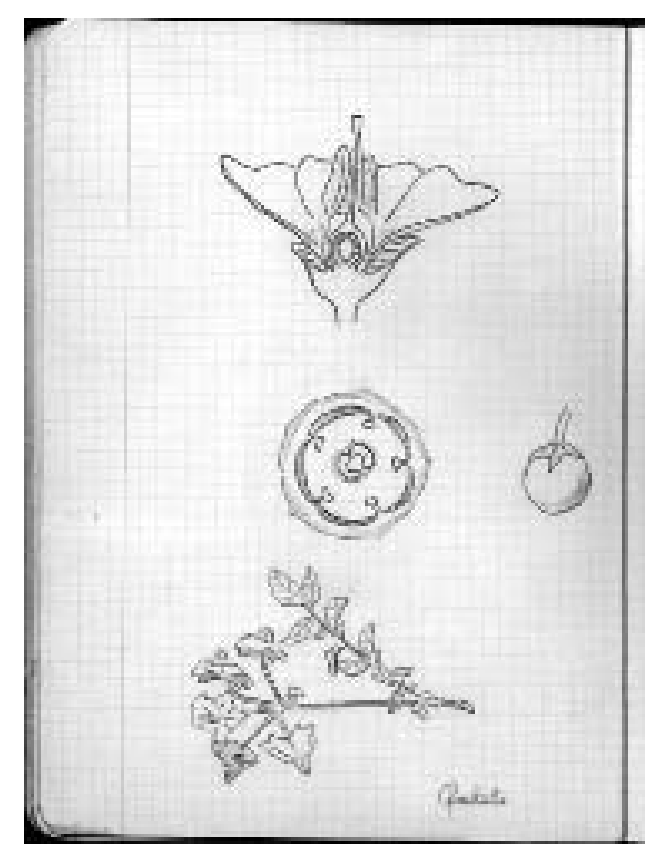

Cuaderno de Agricultura del alumno Javier Cabañas. Instituto-Escuela Sección Retiro.

En el laboratorio de Agricultura, los alumnos contaban con modelos didácticos de botánica del doctor Auzoux (n. ${ }^{\circ}$ registro: 553-555), en papel maché, como los de la espiguilla, el cariópside y el embrión de germen de trigo, la flor y fruto del guisante, entre otros. Sobre las semillas más comunes, su selección y germinación, buscaban información en libros o en trabajos monográficos, en español

ARBOR Vol. 187749 mayo-junio [2011] 501-511 ISSN: 0210-1963

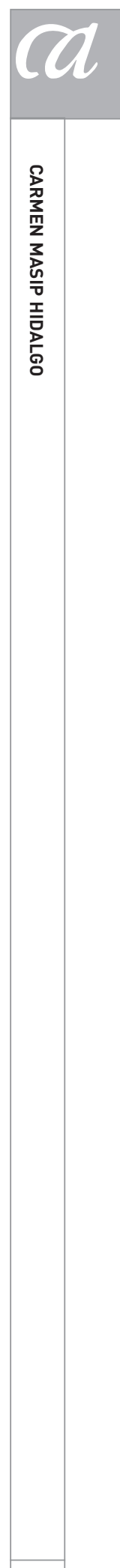

507 
y en otros idiomas. Entre las monografías consultadas, se encontraban la colección del Catecismo del Agricultor o Les graines expliquées, de H. Coupin, que todavia se conservan ${ }^{19}$.

También estudiaban los alumnos la tierra vegetal, el origen, la composición mineralógica, física y biológica y las mejoras de los suelos. Partían para ello de sencillos ensayos sobre el suelo. En los cuadernos (como en el Cuaderno I del alumno Javier Cabañas), se puede comprobar cómo realizaban la granoselección de los componentes en una probeta o su decantación utilizando tubos de ensayo con un embudo y un filtro donde se colocaba la tierra $y_{\text {, al }}$ añadir agua, comprobaban que circulaba según el tamaño de las partículas. En otra práctica, tamizaban el suelo con mallas de distinto diámetro y comprobaban las distintas partes de que se componía y luego dibujaban los resultados en el cuaderno.

Los alumnos estudiaban métodos para analizar las tierras de labor, como los órganoeléctricos, los físicos o los químicos. Con uno de los métodos químicos, por ejemplo, determinaban el contenido de carbonatos sirviéndose del calcímetro de Bernard ${ }^{20}$.

En las clases de Agricultura, Crespí incidía en aspectos que anticipan la moderna ecología, como el considerar algunas plantas como bioindicadores de tipos de suelo. Así, por ejemplo, en uno de los cuadernos (Cuaderno I), aparece anotada la curiosa observación: "De las plantas espontáneas como el pino y los helechos como indicadores de suelos arenosos o las leguminosas espontáneas indicadoras de la tierra caliza". En el laboratorio, proponía a los alumnos experiencias sobre las reacciones químicas entre el suelo y los abonos, como la determinación de las sustancias disueltas en el agua, preguntándoles si contenía nitratos, sulfatos, cloruros, fosfatos, carbonatos, calcio, magnesio, etc. Así determinaban la solubilidad de los abonos. 0 bien les proponía experiencias con tierras desecadas al aire, para demostrar la presencia de sesquióxidos, sílice soluble, hierro y calcio (Cuaderno de Prácticas).

Los cuadernos de los alumnos indican la transmisión de los ensayos más novedosos de la época, precursores de lo que hoy llamamos la lucha biológica, y la importancia de buscar la inmunidad de la planta. Al estudiar la patología vegetal, señalaban ya la defensa contra las plagas con enemigos naturales, es decir, con un método biológico (Cuaderno III) relativamente reciente en la época. Este método consistía en lo que hoy denominamos lucha biológica, uno de los procedimientos más interesantes que hay, aparte de los químicos, para combatir las plagas. Así, por ejemplo, con tal método, se ve en los cuadernos cómo se combatian dos de las enfermedades del naranjo. En Australia, el naranjo no padecía la enfermedad de la cochinilla blanca Icerya purchasi porque allí existía el coleóptero Novius cardinalis, su enemigo natural que acababa con ella. Era la razón por la que se trajeron a Europa miles de ejemplares de Novius cardinales, que se aclimataron y combatieron dicha enfermedad del naranjo, con lo que el problema de la cochinilla se consideró resuelto. También veían los alumnos cómo se podía combatir la enfermedad del naranjo ocasionada por el díptero Ceratitis capitata con un hiperparásito que pone huevos en el interior de la hembra de aquél y acaba destruyéndola. Consideraban igualmente cómo en otros lugares al naranjo no le atacaba la mosca Ceratitis (Cuaderno III) porque desarrollaba inmunidad frente al díptero.

Para estudiar la entomología agrícola, los alumnos disponían de colecciones entomológicas. Se estudiaban sobre todo los insectos que causaban problemas en España (Cuaderno III): la mosca de los cítricos Ceratitis capitata; coleópteros, como el Melolontha vulgaris; ortópteros, como el grillotopo Gryllotalpa; o las langostas con costumbres gregarias más importantes en España, como el Dociostaurus maroccanus y el Acridium peregrinum. Atendian a la descripción y metamorfosis de estos insectos y a los medios de defensa, entre ellos los insecticidas de contacto e ingestión.

El Instituto-Escuela se hizo eco de las ideas de Darwin, lo que no resulta nada extraño tras el empeño que puso la Institución Libre de Enseñanza en la difusión de lo que entonces se denominaba transformismo y en la actualidad evolucionismo (Puelles; Hernández, 2009; Sánchez Ron, 2008, 9; Sequeiros, 2008, 14). Efectivamente, Darwin -que había sido nombrado profesor honorario de la Institución Libre de Enseñanza- no podía faltar ni en la enseñanza de la Agricultura ni en la de la Historia Natural en el Instituto-Escuela. No se olvide que los planteamientos de Darwin impulsaron un nuevo paradigma, el ecológico (López-0cón, 2003, 317) que fue recogido y trasmitido por el Instituto-Escuela. 
Por las anotaciones de los cuadernos de los alumnos, se comprueba que en sus clases (Cuaderno III), Crespí dio a conocer el transformismo e hizo algunas críticas a Lamarck. Para ilustrar el evolucionismo, pone como ejemplo el de unas plantas de valle que al ser llevadas a otra zona de altura modifican sus caracteres, y que al bajarlas de nuevo al valle vuelven a adoptar la forma primitiva. La conclusión del ejemplo es que las variaciones producidas por los cambios de medio no eran hereditarias y que había fenómenos de adaptación en relación con él.

En la materia de Agricultura y de Biología, los alumnos también estudiaban las leyes de Mendel (Cuaderno III) sobre la herencia de los caracteres adquiridos, con varios ejemplos de cruces entre variedades, enana y alta, de plantas de guisante; o entre dos variedades puras de calabaza blanca y discoidal, con variedades amarilla y esférica. Luego analizaban los resultados de los cruces obtenidos en la primera (F1) y en la segunda (F2) generación filial. Al estudiar el ganado y analizar las principales causas de variación de las especies y los medios de selección y mejora del ganado o la especiación en Zootecnia, se señala en los cuadernos que la característica de una especie reside en los cromosomas y que algunos caracteres dentro de las especies no son hereditarios y que dependen del medio. También se indica que el carácter de especie entre carácter máximo y mínimo tiene que estar en los cromosomas de las células y cada carácter tiene una representación en el cromosoma (Cuaderno III). En cuanto a la selección de los animales (Cuaderno IV) y plantas, los cuadernos explican cómo se seleccionaban los caracteres de interés.

\section{Conclusiones}

Para concluir este breve recorrido por la trayectoria docente de Luis Crespí, debo llamar la atención sobre el tesoro documental que suponen para la historia de la educación en España los cuadernos de clase y de trabajo de los alumnos del Instituto-Escuela que se han conservado. Los cuadernos de Agricultura de Luis Crespí, en particular, aportan una valiosísima información acerca de la pedagogía innovadora y los contenidos actualizados que informaron su ejemplar labor docente en su etapa como catedrático de Agricultura del Instituto-Escuela. Algunos de sus alumnos de entonces, como Julio Caro Baroja o Josefina Calandre (Calandre, 2008,60), recuerdan con admiración la excelente formación que recibieron en sus clases. Con sentidas palabras de agradecimiento, Julio Caro Baroja (Caro Baroja, 1986, 150) lo describe asi:

Fue éste un profesor de Agricultura, don Luis Crespi, naturalista distinguido, hombre alto, sardónico, muy buen profesor [...]. Muchos años después pasada la guerra, veía yo a don Luis alguna vez y me franqueó notas y dibujos acerca de los aperos, cultivos, etc. También me contó cómo habia acompañado a Vavílov, creo que por Asturias.

Con este trabajo, he querido sumarme a todos aquellas personas que en los últimos años han contribuido y siguen contribuyendo con sus estudios e investigaciones a resca-

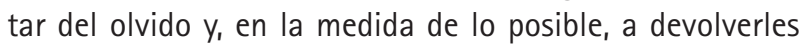
la palabra a tantas y tantas personalidades del ámbito científico de nuestra Edad de Plata, una de las cuales fue Luis Crespí Jaume, catedrático del Instituto-Escuela de Madrid y científico de la JAE.

\section{NOTAS}

Recibido: 1 de marzo de 2010

Aceptado: 30 de junio de 2010
1 Archivo JAE Edad de Plata.

2 lbidem.

3 lbidem.

4 Gaceta de Madrid 04/08/1922, n. ${ }^{\circ}$ 316, p. 692, y Archivo del IES Brianda de Mendoza, de Guadalajara.

5 Archivo del IES Brianda de Mendoza.
6 Gaceta de Madrid, 11/11/1924, n. 316 , p. 692 , y 24/11/1924, n. ${ }^{\circ} 329$, p.890.

7 Actualmente en el Herbario del RJB de Madrid se conserva la colección de líquenes de Crespí que fue enriqueciendo durante años hasta llegar a 1.078 ejemplares.

8 Archivo MNCN: ACN0285/009.

9 Archivo MNCN: ACN 285/014. 
10 Gaceta de Madrid, 23/04/1930, n. 113, p. 470.

11 Archivo Clases Pasivas.

12 Archivo MNCN: ACN272/005.

13 Archivo de Clases Pasivas.

14 Memorias JAE curso 1923-24, pp. 346-347.

15 Biblioteca Histórica del IES Isabel la Católica.

16 Todos los cuadernos de clase (I-IIIII-IV) y de prácticas de Agricultura, a los que se hace referencia en este artículo pertenecen a la colección de Javier Cabañas, alumno del Instituto-Escuela Sección Retiro, 5. Grado, Profesor Luis Crespí. Curso 1933-34.

17 Todo el material científico al que se hace referencia en este artículo fue del Instituto-Escuela Sección Retiro y actualmente se encuentra en el IES Isabel la Católica. En cada pieza se hace referencia al n. ${ }^{\circ}$ de catálogo. En este caso se refiere al n. ${ }^{\circ} 603$.

18 Biblioteca Histórica. IES Isabel la Católica.

19 Biblioteca Histórica IES Isabel la Católica.

20 El método se basa en la reacción del ácido clorhídrico $(\mathrm{HCl})$ con el carbonato cálcico $\left(\mathrm{CaCO}_{3}\right)$ con desprendimiento de anhídrido carbónico $\left(\mathrm{CO}_{2}\right)$. Este gas desplaza un volumen de un líquido indicador que se cuantifica.

\section{BIBLIOGRAFÍA}

Baratas Díaz, Alfredo (1998): "La Ciencia española ante la crisis del 98: semillas, frutos y agostamiento", Cuadernos de Historia Contemporánea, n. ${ }^{\circ}$ 20, Madrid, UCM, pp. 151-163.

Bernal Martínez, José Mariano y Comas Rubi, Francesca (2006): "La función social de las Ciencias de la Naturaleza: Una influencia europea en el currículum escolar en España", Sala- manca, Ed. Universidad de Salamanca, pp. 131-156.

Calandre Hoenigsfeld, Cristina (2008): "Josefina Calandre", Observación y experimentación en las Ciencias $\mathrm{Na}$ turales, enero, pp. 60-69, Madrid. "ESTUDI0" Boletín de actividades 14 Curso 2007/2008.

Casado de Otaola, Santos (2000): Los primeros pasos de la ecología en España, Madrid, Ministerio de Medio Ambiente, Organismo Autónomo de Parques Nacionales.

Caro Baroja, Julio (1986): Los Baroja, Madrid, Taurus.

Crespi Jaume, Luis (1915): "Las anomalías florales del maíz y los trabajos de Blainghem", Bol. R.S.E.H.N., Madrid, Real Sociedad Española de Historia Natural, pp. 259-268.

- (1922): La soja y su cultivo en España, Catecismo del agricultor y ganadero, n. ${ }^{\circ}$ 62, Madrid, Ed. Calpe.

- (1929): "Notas sobre una geografía botánica en Galicia", Mem. S.E.H.N., Madrid, Real Sociedad Española de Historia Natural, pp. 799-810.

- (1929): "Contribución al folklore gaIlego", Conf. Res. Ci. S.E.H.N., Madrid, Real Sociedad Española de Historia Natural, pp. 4:5-19.

Crespí, Antonio L. y González Bueno, Antonio (1990): "Luis Crespí Jaume (Madrid, 1889-Madrid, 1963)", Acta de Botánica Malacitana, n. 15, Málaga, UMA, pp. 341-345.

Crespi Jaume, Luis e Iglesias, Luis (1929): "Los prados de las regiones media y montaña de Galicia", Bol. S.E.H.N., Madrid, Real Sociedad Española de Historia Natural, pp. 127-142.

Fraga Vázquez, Xosé A. (1995): "Historia do Instituto de Pontevedra. As Ciencias Naturales, 1845-1936", Pontenova, Revista de Investigación Xove, n. ${ }^{\circ}$, Pontevedra, Diputación Provincial de Pontevedra.
Fraga, Xosé A. y Gurriarán R. (2007): "Galicia y la JAE", Tiempos de investigación JAE-CSIC. Cien años de ciencia en España, Madrid, CSIC, pp. 187-195.

González Bueno, Antonio (1993): "Herbarios, colectores e indices de semillas como fuentes para la historia de las ciencias", Madrid, El Ateneo, pp. 2836.

JAE (1925): Un ensayo pedagógico. El Instituto-Escuela de Segunda Enseñanza de Madrid (Organización, métodos y resultados), Madrid, JAE, pp. 284287.

J. Rico, Victor (1999): "Aspicilia crespiana, A New Lichen Species From Southern Europe", Lichenologist, 31(2): 129139. Article No.lich.1998.08. Londres. The British Lichen Society. http:// www.idealibrary.com.

López-Ocón Cabrera, Leoncio (2003): Breve historia de la ciencia española, Madrid, Alianza Editorial.

Martínez Alfaro, Encarnación (2009): Un laboratorio pedagógico de la Junta para Ampliación de Estudios. El Instituto-Escuela Sección Retiro de Madrid, Madrid, Biblioteca Nueva/ CEIMES.

Puelles, Manuel y Hernández, Margarita (2009): "El darwinismo en los manuales escolares de ciencias naturales de segunda enseñanza desde la publicación del Origen de las especies en España hasta finales del s. XIX", Anuario de Historia de la Iglesia XVIII, Universidad de Navarra, pp. 69-83.

Sánchez de Andrés, Leticia (2007): "Los cuadernos escolares. Una herramienta para la renovación pedagógica", El Laboratorio de España. La Junta para Ampliación de Estudios e Investigaciones Científicas 1907-1939, Madrid, CSIC, pp. 421-428.

Sampaio, Gonzalo y Crespí, Luis (1927): "Los líquenes de la provincia de Pontevedra", Bol. Soc. Esp. Hist. Nat., 27: 
136-151, Madrid, Real Sociedad Española de Historia Natural.

Sánchez Ron, José Manuel (2008): "Introducción", Boletín de la Institución Libre de Enseñanza, n. ${ }^{\text {s. }}$. 1-72, octubre, Madrid, Fundación Francisco Giner de los Ríos.
Sequeiros San Román, Leandro (2008): "El debate sobre el transformismo de Darwin hace 150 años y en la actualidad", Presentación del facsímil Estudio sobre el transformismo, por Don Rafael Garcia Álvarez, Granada, Instituto Padre Suárez, pp. 1-34.
Vavilov, Nicolái Ivánovich (1931): "Expediciones por España", II Congreso Internacional de Historia de la Ciencia, Londres. Traducción española de Pablo Huerga Melcón, El Catoblepas, n. ${ }^{\circ}$ 38, 2005. http://www.nodulo.org/ ec/2004/n032p17.htm. 